\title{
Left Ventricle Contouring in Cardiac Images Based on Deep Reinforcement Learning
}

This paper was downloaded from TechRxiv (https://www.techrxiv.org).

\section{LICENSE}

CC BY 4.0

SUBMISSION DATE / POSTED DATE

$26-07-2021$ / 27-07-2021

\section{CITATION}

Yin, Sixing; Han, Yameng; Pan, Judong; Wang, YIning; Li, Shufang; Yu, F. Richard (2021): Left Ventricle Contouring in Cardiac Images Based on Deep Reinforcement Learning. TechRxiv. Preprint. https://doi.org/10.36227/techrxiv.15052428.v1

$\mathrm{DOI}$

10.36227/techrxiv.15052428.v1 


\title{
Left Ventricle Contouring in Cardiac Images Based on Deep Reinforcement Learning
}

\author{
Sixing Yin, Member, IEEE, Yameng Han, Judong Pan, Yining Wang, Shufang Li, Senior Member, IEEE \\ and F. Richard Yu, Fellow, IEEE
}

\begin{abstract}
Assessment of the left ventricle segmentation in cardiac magnetic resonance imaging (MRI) is of crucial importance for cardiac disease diagnosis. However, conventional manual segmentation is a tedious task that requires excessive human effort, which makes automated segmentation highly desirable in practice to facilitate the process of clinical diagnosis. In this paper, we propose a novel reinforcement-learning-based framework for left ventricle contouring, which mimics how a cardiologist outlines the left ventricle in a cardiac image. Since such a contour drawing process is simply moving a paintbrush along a specific trajectory, it is thus analogized to a path finding problem. Following the algorithm of proximal policy optimization (PPO), we train a policy network, which makes a stochastic decision on the agent's movement according to its local observation such that the generated trajectory matches the true contour of the left ventricle as much as possible. Moreover, we design a deep learning model with a customized loss function to generate the agent's landing spot (or coordinate of its initial position on a cardiac image). The experiment results show that the coordinate of the generated landing spot is sufficiently close to the true contour and the proposed reinforcement-learning-based approach outperforms the existing U-net model even with limited training set.
\end{abstract}

Index Terms-Left ventricle contouring, cardiac image segmentation, deep reinforcement learning

\section{INTRODUCTION}

According to the recent statistics from American heart association (AHA), cardiovascular diseases has been the top leading cause of death globally [1]. Magnetic resonance imaging (MRI) is a non-invasive imaging technology, which can objectively and accurately depict the tissue structure, pathological changes, and therefore provide important information for not only clinicians but also for biomedical researchers. Specially, cardiac MRI generally has been considered as the

Sixing Yin, Yameng $\mathrm{Han}$ and Shufang $\mathrm{Li}$ are with Beijing Key Laboratory of Network System Architecture and Convergence, Beijing University of Posts and Telecommunications, Beijing 100876, China (e-mail:yinsixing@bupt.edu.cn, han_1997@bupt.edu.cn, lisf@bupt.edu.cn).

Judong Pan is with University of California San Francisco, San Francisco, CA 94117, The United States (e-mail: judong.pan@ucsf.edu).

Yining Wang is with State Key Laboratory of Complex Severe and Rare Diseases, Chinese Academy of Medical Sciences and Peking Union Medical College, Beijing 100730, China (email:yiningpumc@163.com).

F. Richard $\mathrm{Yu}$ is with the Department of Systems and Computer Engineering, Carleton University, Ottawa, ON K1S 5B6, Canada (e-mail: richard.yu@carleton.ca).
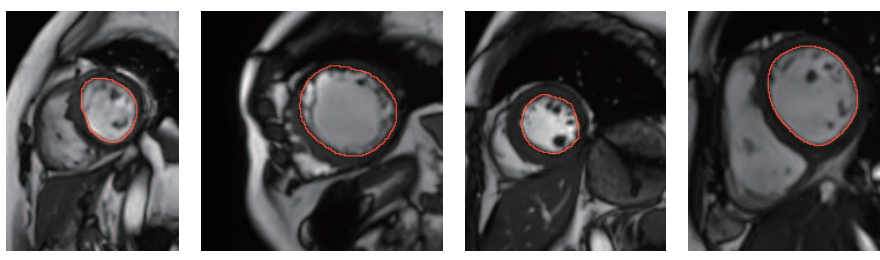

Fig. 1. Examples of left ventricle contouring in cardiac images.

"gold standard" for measuring essential physical parameters (e.g., myocardial mass, end-diastolic volume and ejection fraction) to identify a wide range of cardiovascular diseases [2].

In cardiac MRI analysis, assessment of the left ventricle segmentation is of crucial importance for diagnosis of cardiac pathologies, including coronary heart disease dysplasia and cardiomyopathies [3], and thus has drawn increasing attention over the past decade. Normally, left ventricle segmentation is manually carried out by human experts and is time-consuming with tedious effort, especially when dealing with large-scale datasets. Therefore, techniques for automating left ventricle segmentation in cardiac MRI become increasingly imperative so as to facilitate the process of clinical diagnosis. Meanwhile, it also necessitates sufficient accuracy since an incorrect segmentation can mislead clinicians to draw incorrect conclusions from the imaging data, which could result in devastating consequences.

In recent years, deep learning has received extensive attention and achieved tremendous success in a great number of areas since deep-learning-based models are capable of capturing more complicated features hidden in large-scale datasets [4]. Tremendous effort has been made to automate medical imaging analysis and facilitate diagnosis through deep learning [5]. As a prevailing computational structure for deep learning models, convolutional neural networks (CNNs), as well as its derivative variants such as U-net [6] and fully convolutional networks (FCNs) [7] have achieved state-of-theart performance in medical image segmentation [8]. However, due to the inevitable challenges of medical images, such as poor image quality, different imaging and segmentation protocols, and random variation among patients, such deeplearning-based approaches have still been considered untrustworthy and thus not yet been extensively put into clinical use. Such untrustworthiness comes mainly from the fact that the hidden features extracted by those end-to-end models based on 
deep learning fall short of human-level understandability and explainability, which inevitably leads to potential high risk in clinical trials.

In this sense, a model that works in accordance with how human experts segment medical images is more preferable in practice. In contrast with most of the existing deep learning models that identify an object of interest in an image through pixel-level classification, a human expert basically focuses on contouring an object in a medical image instead, which is also the route work of a specialist doctor to provide explicit clue of clinical diagnosis. Fig. 1 shows cardiac image examples from four patients along with contours of the left ventricles. It can be visually found that due to existence of the fuzzy edges and irregular cavities, outlining the left ventricle in a cardiac image with machine-learning-based approaches is a non-trivial task.

With such motivation, in this paper, we propose a novel approach for left ventricle contouring based on reinforcement learning [9], which mimics how a cardiologist outlines the left ventricle in a cardiac image. The idea is based on the fact that the contour drawing process is simply moving a paintbrush along a specific trajectory and thus can be analogized to a path finding problem. The overall framework of the proposed approach for left ventricle contouring is shown in Fig. 2, where a robot tries to make the best movement on a cardiac image according to its local observation such that the trajectory it walks along sufficiently matches the contour of the left ventricle (the reinforcement-learning-based framework in Fig. 2 will be further interpreted with more detail in Section III). The contributions of this paper are summarized as follows:

(1) By analogizing left ventricle contouring to a path finding problem, we propose a a novel reinforcement-learning-based framework for left ventricle contouring, the mechanism of which is different from most the existing supervised-learningbased approaches.

(2) By defining the problem of left ventricle contouring under the framework of reinforcement learning, we train a policy network that takes the agent's local observation as input and outputs a stochastic movement such that the generated trajectory matches the true contour of the left ventricle as much as possible.

(3) In order for the practicality of the proposed approach, we design a deep learning model with a customized loss function to generate the agent's landing spot, i.e., a coordinate on the cardiac image, from which the agent makes its first movement.

(4) Through comprehensive experiments, we show that the coordinate of the generated landing spot is sufficiently close to the true contour and the proposed reinforcement-learningbased approach outperforms the existing U-net model even with limited training set.

The rest of this paper is organized as follows. Related work is reviewed in Section II. The proposed reinforcementlearning-based approach is elaborated in Section III. Experimental results are presented along with analysis in Section IV. We conclude the whole paper in Section V.

\section{RELATED WORK}

\section{A. Conventional Image Segmentation}

As a commonly used technique in digital image processing and analysis, image segmentation is to partition an image into multiple parts or regions, which contain pixels with similar attributes. Research on conventional image segmentation can be categorized into three classes: edge-based segmentation, threshold-based (intensity-based) segmentation and regionbased segmentation [10]. With threshold-based segmentation, one or multiple thresholds are computed according to certain standards (e.g., local characteristics). Though computationally simple and efficient, threshold-based segmentation could result in object coherency in existence of indistinct edges. Regionbased segmentation groups pixels with similar property by utilizing local spatial information such that it performs better in handling spatial discontinuity while "over-segmentation" or "under-segmentation" could be a potential issue with inappropriate threshold setting. Edge-based segmentation detects edges between different regions through certain computations (e.g., derivative operator) and thus is sensitive to strong noise and excessive smooth boundaries [11].

\section{B. Deep-learning-based Image Segmentation}

Over the past few years, due to automatic feature extraction, robustness to natural variation as well as large-scale parallel computation, deep learning has increasingly received attention from both industry and academia and has achieved remarkable success in a wide range of computer vision applications [12], among which there have been a great number of works focus on performance improvement for image segmentation with carefully designed computational models. Under the framework of deep learning, image segmentation can be formulated as a pixel-level classification problem, which predicts the single label of each pixel rather than the overall label of the image. With deep learning, an end-to-end computational model can be designed such that image segmentation can be done by simply feeding an image into the model. Due to the excellent performance on popular benchmarks, the deep learning models has led to a paradigm shift in image segmentation.

As the most successful computational structure in the deep learning community, convolutional neural networks (CNNs) has made breakthrough in a variety of applications for the recent years, especially in computer vision tasks, and most of the existing leading-edge deep learning structures and models for image segmentation originates from CNNs [13]. To integrate more context, probabilistic graphical models such as conditional random fields are incorporated into CNNs and relevant models and algorithms have achieved encouraging results [14] [15].

In 2015, Long et al. proposed the fully cnvolutional networks (FCNs) for semantic image segmentation [7]. Including only convolutional layers, the FCN takes images with arbitrary size as input and outputs the segmentation map with the same size. However, since FCN-based models necessitate transposed convolution (also known as deconvolution) and up-sampling to output the segmentation map with the same size of the input image, the extracted features could be relatively sparse, which makes the segmentation result less refined. Moreover, 
FCN-based models ignores global context information. In response to this, the encoder-decoder model is proposed for image segmentation, where the encoder down-samples the input image to generate a feature map with lower resolution (which usually adopts existing structures for feature extraction, e.g., part of the VGG-16 network) and the decoder up-samples the feature map and restores the segmentation map [16].

Originally invented mainly for medical image segmentation, U-net [6] and V-net [17] are two popular computational structures that are inspired by FCNs and the encoder-decoder model. The U-net structure comprises a contracting (downsampling) path to capture context and a expanding (upsampling) path that symmetrically concatenates each layer of the contracting path to enable precise localization. The U-net has achieved outstanding performance for image segmentation in cell tracking challenge 2015 and there have been a great number of variants [18] [19] [20]. With a similar structure, Vnet is another well-known FCN-based model for 3D medical image with Dice score as the loss function. There have been also improved versions of V-net, e.g., the progressive dense V-net (PDV-net) that combines dense V-net and progressive holistically-nested network is proposed for efficient automatic segmentation of pulmonary lobes from chest CT images [21], and a lightweight V-net (LV-Net) with inverted residual bottleneck and 3D average pooling is proposed for liver image segmentation [22].

\section{Summary}

Most of the existing approaches for conventional image segmentation follows routine procedures and requires human intervene and expertise in computer vision, which limits their popularity. On the contrary, under the deep learning paradigm, image segmentation is implemented through an end-to-end computational model, which can be parametrized with prevailing structures such as CNNs and FCNs. Since image segmentation can be simply considered as a pixel-level classification problem, deep learning models can be efficiently trained with existing gradient-based methods, which makes expertise no longer a prerequisite. In general, however, most of the existing work on deep-learning-based image segmentation focus on design for the end-to-end computational model so as to improve segmentation performance. Therefore, though with eye-catching performance on benchmarks, most of the deep learning models for medical image segmentation are still at the prototype stage and have not yet put into clinical use due to limited understandability and explainability. Motivated by this, we seek to design a model that mimics how human experts identify an object of interest in an image.

\section{Methodology}

\section{A. Data Preprocessing}

Our entire dataset contains two-dimensional cardiac images acquired through magnetic resonance imaging (MRI) with non-uniform sizes. For each image, the left ventricle has been outlined by an experienced cardiologist and an associated binary image with the same size has been generated as the contour image, where pixels located on the contour of the left

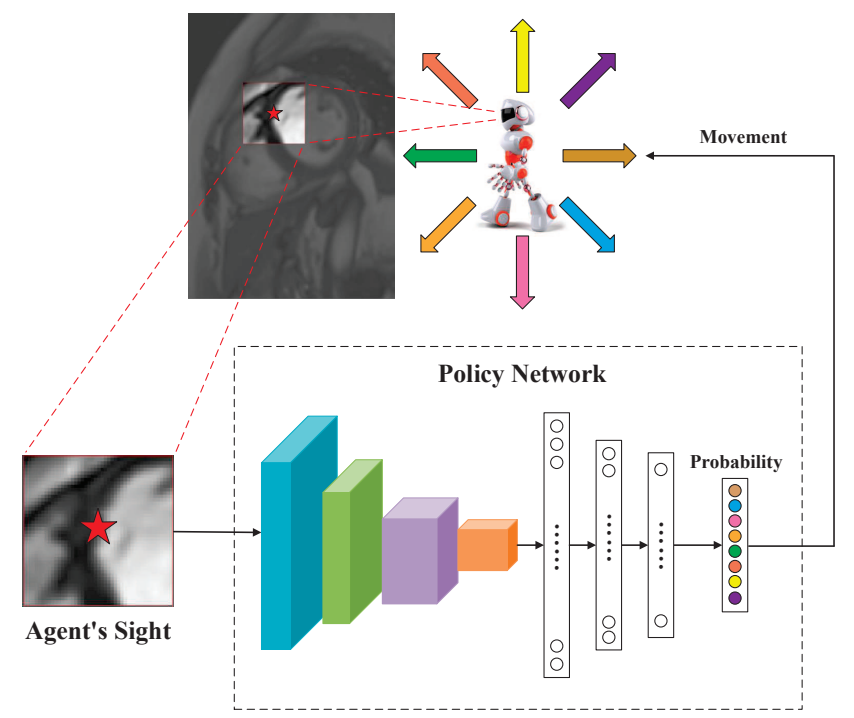

Fig. 2. The overall framework of left ventricle contouring based on reinforcement learning.

ventricle marked by the cardiologist have value 1 while the remaining pixels have value 0 .

We consider each contour of the left ventricle as a sequential point set, which are counter-clockwise sorted without loss of generality. In the original dataset, the contours marked by the cardiologists are commonly discontinuous "trajectories", i.e., two consecutive points on a contour probably fall outside the neighbourhood (a $3 \times 3$ patch centered by one pixel) of one another. In order to cater for the reinforcement learning setting defined in the next subsection, we have to first refine the original contours by interpolating sufficient extra points between two consecutive ones that are non-adjacent to each other to meet the continuity requirement, i.e., any point on a contour always have neighbours located within its $3 \times 3$ patch. After thoroughly inspecting all the contour images, we find that points on the original contours are densely located in vertical direction (i.e., two consecutive points are always located on two adjacent rows) while scarcely located in horizontal direction (i.e., two consecutive points are rarely located on two adjacent columns). Therefore, we can simply scan every two points on a contour and place extra points on every pixel on the row spacing between the two points.

\section{B. Problem Definition}

Instead of supervised-learning-based image segmentation to highlight the left ventricle, we focus on human-like contouring that mimics how a cardiologist outlines the left ventricle in a cardiac image. Since such a contour drawing process is simply moving a paintbrush along a specific trajectory, it can be analogized to a path finding problem, where a robot explores the unseen world and tries to find its way along the object boundary. In this sense, contouring the left ventricle in a cardiac image can be translated into a reinforcement learning problem, where an agent seeks to make the best decision, i.e., a single-step movement, by interacting with an environment so as to travel as closely as possible to the true contour. Under 
the reinforcement learning framework, a policy network is trained to output the best movement according to the agent's local observation, as shown in Fig. 2. In the following part, the key elements of reinforcement learning framework for left ventricle contouring are formally defined.

1) Environment: An environment in left ventricle contouring can be defined as any cardiac image and its associated contour image. Here the whole cardiac image and the true contour of the left ventricle are unseen by the agent. Every time the agent interacts with such an environment by taking a single action, a reward is given by the environment as the feedback to indicate how good that action was, based on which the agent further updates its policy for a better decision, i.e., moving closer to the true contour.

2) State: In the reinforcement learning setting, a state is what the agent can observe, and based upon on which it can choose an appropriate action. In this paper, we specify that, being located at any pixel in a cardiac image, the agent can only myopically observe its surrounding sight rather than the whole image. This is an analogy to a path-finding robot with limited eyesight. We define the state $s$ as the $N \times N$ square patch centered by the pixel where agent is located. One advantage of such setting is that the policy network can be designed with a uniform structure since the input size is always $N \times N$.

3) Action: Since we analogize left ventricle contouring in a cardiac image to path finding in an unseen world, the agent's one-step movement can be defined as the action. Here we specify that at each step, the agent takes a one-pixel move towards one of its eight outbound direction and moves to one of its eight neighbouring pixels. Thus, the action $a$ with such definition is discrete and can be numbered with $a \in\{1, \ldots, 8\}$. Then with the agent's surrounding sight defined as state, Markovian property holds for state transition at each step, i.e., the next state depends only on the current one and the action taken by the agent.

4) Reward and Return: At each step, the environment gives a reward back to the agent based on its action. Since it is more desirable for the agent to stay as close as possible to the true contour while travelling, reward (denoted by $r_{t}$ for step $t$ ) can be defined as the negative distance between the agent's current position (denoted by $p_{t}$ ) and the corresponding position on the true contour (denoted by $p_{t}^{\prime}$ ), i.e., $r_{t}=-\left|p_{t}-p_{t}^{\prime}\right|$. Therefore, the discounted accumulated return from step $t$ to the end of the episode (also termed "reward-to-go") can be defined as the discounted sum of negative distance over the entire episode, i.e.,

$$
R_{t}=-\sum_{\tau=t}^{T} \gamma^{\tau-t}\left|p_{\tau}-p_{\tau}^{\prime}\right|,
$$

where $\gamma \in(0,1]$ and $T$ denote the discount factor and number of steps in one episode (which differs in different cardiac images), respectively.

5) Episode Termination: In reinforcement learning framework, episode termination indicator is important as well. Normally, an episode can be terminated once the agent has finished the entire steps of actions, the total number of which is equal to the length of the true contour. However, an exception is that the agent might have moved illegitimately outside the cardiac image before the episode ends normally, which is a common case at the early stage of model training when the agent is not sufficiently "smart" to follow the true contour. In this case, we specify that the episode is terminated in advance and a reward of an extremely small negative value is given to the agent as a penalty for the illegal movement.

\section{Policy Optimization}

The previous definition for the elements of reinforcement learning implies a stochastic policy for left ventricle contouring in a cardiac image, i.e., the agent chooses its movement according to a probability distribution, which is output by the policy network with the agent's surrounding sight as input. We resort to proximal policy optimization (PPO) as the training algorithm: a policy-based algorithm which aims at iteratively improving the policy with a relatively small update [23]. With the PPO algorithm, the policy network is iteratively updated to maximize a clipped surrogate objective function defined as

$$
\begin{aligned}
L(\theta)= & \mathbb{E}_{s, a \sim \pi_{\theta_{k}}}\left[\operatorname { m i n } \left(\frac{\pi_{\theta}(a \mid s)}{\pi_{\theta_{k}}(a \mid s)} A^{\pi_{\theta_{k}}}(s, a),\right.\right. \\
& \left.\left.\operatorname{CLIP}\left(\frac{\pi_{\theta}(a \mid s)}{\pi_{\theta_{k}}(a \mid s)}, 1-\epsilon, 1+\epsilon\right) A^{\pi_{\theta_{k}}}(s, a)\right)\right]
\end{aligned}
$$

for iteration $k$, where $\pi_{\theta}$ denote the policy under parameter $\theta, A^{\pi}(s, a)$ refers to the advantage of action $a$ for state $s$ under policy $\pi, \epsilon$ is a hyper-parameter that ranges from 0 to 1 to control the deviation from the current policy $\pi_{\theta_{k}}$ to the updated one $\pi_{\theta}$ and the clip function $\operatorname{CLIP}(., l, u)$ is defined as

$$
\operatorname{CLIP}(x, l, u)=\left\{\begin{array}{l}
l, x<l \\
x, l \leq x \leq u \\
u, x>u
\end{array}\right.
$$

The PPO algorithm for left ventricle contouring is summarized in Algorithm 1, where $T_{i}$ refers to the total number of steps in episode trajectory $\tau_{i}$, i.e., the contour length. Here the stochastic policy for choosing a movement is trained in an on-policy manner following the "actor-critic" framework in policy-based algorithms. In each iteration, one episode is run according to the up-to-date policy for each training image such that a batch of experience trajectories are collected as training samples, based on which the advantage is estimated. Even with limited training dataset, diversity of the collected episode trajectories can still be guaranteed by randomly choosing the agent's starting point on a contour in each iteration. Then the parameters of the policy network $\theta$ and value network $\phi$ are alternately updated to maximize the surrogate objective function in (2) ("actor" update) and minimize the meansquare-error between the estimated reward-to-go and the one predicted by the value network ("critic" update) via stochastic gradient ascent/descent.

\section{Generating Landing Spot}

Following Algorithm 1, the agent's policy can be iteratively improved such that it is likely capable of moving closer to the true contour after rounds of training. During training, the 


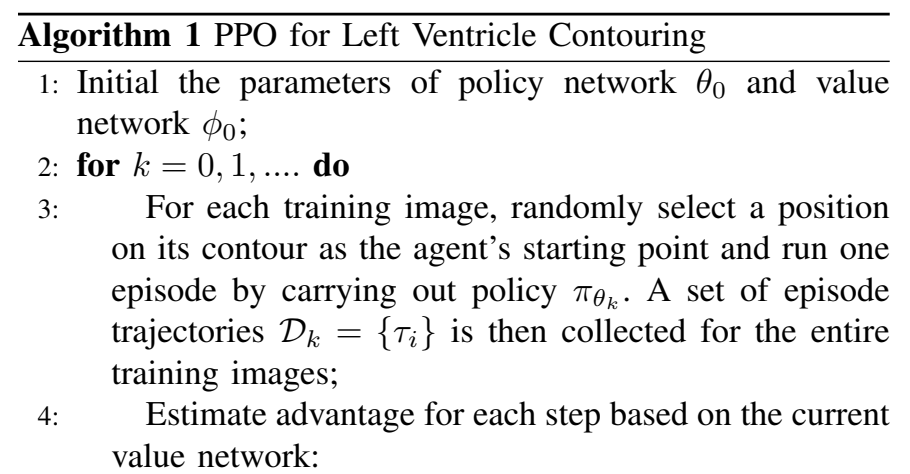

$$
A\left(s_{t}, a_{t}\right)=\sum_{t^{\prime}=t}^{T} \gamma^{t^{\prime}-t} r_{t^{\prime}}-V_{\phi_{k}}\left(s_{t}\right)
$$

5: Update the parameters of the policy network to maximize the mean surrogate objective defined in (2):

$$
\theta_{k+1}=\arg \max _{\theta} \frac{1}{\left|\mathcal{D}_{k}\right|} \sum_{\tau_{i} \in \mathcal{D}_{k}} \frac{1}{T_{i}} \sum_{t=0}^{T_{i}} L(\theta)
$$

6: Update the parameters of the value network to minimize the mean squared error between the predicted and estimated value functions:

$$
\phi_{k+1}=\arg \min _{\phi} \frac{1}{\left|\mathcal{D}_{k}\right|} \sum_{\tau_{i} \in \mathcal{D}_{k}} \frac{1}{T_{i}} \sum_{t=0}^{T_{i}}\left(V_{\phi_{k}}\left(s_{t}\right)-\sum_{t^{\prime}=t}^{T} \gamma^{t^{\prime}-t} r_{t^{\prime}}\right.
$$

7: end for

agent's starting point is randomly chosen on a true contour. However, this is unrealistic for testing stage since the true contour of an unseen cardiac image is inaccessible. Therefore, where the agent should start to move on an unseen cardiac image still needs to be figured out. Apparently, the agent's landing spot should be as close to the true contour as possible. Otherwise, if the landing spot is excessively far away from the true contour, it is difficult for the agent to be back on track since its surrounding sight in this case could be significantly different from what it observed during training.

Intuitively, a deep learning model can be trained to generate the coordinate of the agent's appropriate landing spot. After inspecting all the cardiac images, we found that part of true contours always falls into the upper-right quadrant without exception. Therefore, we only focus on the upper-right subimage with uniform size for each cardiac image. The landing spot generator then takes a sub-image as input and is expected to output a coordinate that is as close to any point on the true contour as possible. This can be done through training a supervised-learning model with the loss function defined as the mean minimum distance between the output coordinate and that of any point on the true contour:

$$
L(\psi)=\frac{1}{B} \sum_{i=1}^{B} \min \left(d_{1}, \ldots, d_{T_{i}}\right),
$$

where

$$
d_{t}=\left|G_{\psi}(x)-p_{t}^{\prime}\right|
$$

refers to the distance between the output coordinate and the $t$ th point $p_{t}^{\prime}$ on the true contour of a sub-image, $G_{\psi}(x)$ refers to the landing spot generator with $\psi$ as parameters and sub-image $x$ as input, $T_{i}$ denotes the total length of the true contour that falls into the $i$-th sub-image and $B$ denotes the batch size. In model training, due to the small size of our training set (32 as descried in Section IV-A), the entire training set instead of a batch (a small portion) can be fed into the model in each iteration of parameter update. Therefore, we resort to standard gradient descent to training the model instead of the stochastic gradient descent: instead of being predefined as a static hyperparameter, the learning rate is optimized through line search, e.g., the secant method. Specifically, in iteration $k$ of model training, the optimal learning rate $\lambda_{k}$ is determined by solving

$$
\lambda_{k}=\arg \min _{\lambda>0} L\left(\psi_{k}-\lambda \nabla L\left(\psi_{k}\right)\right) .
$$

The algorithm for training the landing spot generator is summarized in Algorithm 2. Since only a sub-image with smaller size is required as the input and a small portion of a true contour is involved in the loss function, the landing spot generator adapts to different sizes of cardiac images and the training efficiency can be significantly enhanced even with limited training dataset.

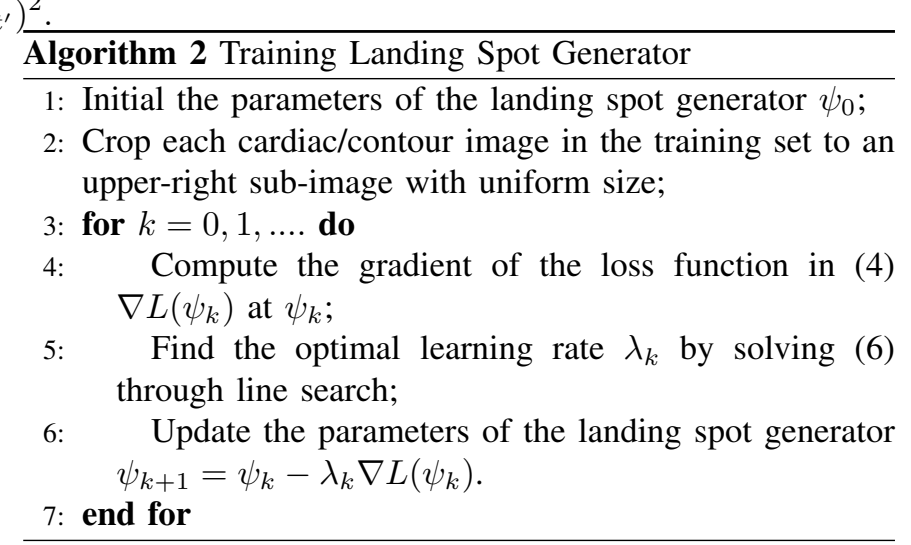

The output coordinate of the landing spot generator is not necessarily an integer one, which is probably the case. Therefore, we simply round the output coordinate to its nearest integer in the sub-image. Moreover, the output coordinate still has to be translated back to that in the original (fullsized) cardiac image. This can be done by simply leaving the vertical coordinate unchanged while changing the horizontal coordinate $x$ following

$$
x \Leftarrow x+\left(N_{x}-W\right),
$$

where $N_{x}$ refers to the horizontal size of the original cardiac image and $W$ denotes the width of the sub-image.

\section{EXPERIMENT}

\section{A. Dataset}

Our dataset is provided by the radiology department of a "triple-A-level" hospital in Beijing, China. It contains 100 twodimensional MRI cardiac images in total, which was collected 
from 60 patients, and has non-uniform sizes of $208 \times 138$, $208 \times 150,208 \times 162,208 \times 168,208 \times 186$ and $208 \times 210$, respectively. Each cardiac image is associated with a contour image created by an experienced radiologists subspecialized in cardiovascular imaging as the ground truth. Throughout the experiment, 32 images are selected as the training set, 8 as the validation set while the remaining 60 as the testing set. All the images are normalized with pixel values from 0 to 1 before being fed into the model.

\section{B. Model Design}

In the study, we assumed that the agent could only observe its surrounding sight within a $21 \times 21$ patch, which serves as the input of the policy network. The network design of the policy network is given in Table I, which contains two convolution-pooling layers followed by three fully connected (FC) layers. All the layers except the last one adopts the "Relu" activation function. Since the policy network outputs a discrete probability distribution, which corresponds to the probability of moving towards one of the eight outbound directions with one-pixel step, the policy network ends with a "softmax" that outputs eight probabilities.

TABLE I

Policy Network StRucture

\begin{tabular}{|c|c|c|}
\hline Layer & Filter size & stride \\
\hline Conv2D-16 & $5 \times 5$ & $1 \times 1$ \\
\hline Max_pooling2D & $2 \times 2$ & $2 \times 2$ \\
\hline Conv2D-64 & $3 \times 3$ & $1 \times 1$ \\
\hline Max_pooling2D & $2 \times 2$ & $2 \times 2$ \\
\hline \multicolumn{3}{|c|}{ FC-256 } \\
\hline \multicolumn{3}{|c|}{ FC-64 } \\
\hline & FC-8 \\
\hline
\end{tabular}

Similarly, the value network also takes the agent's surrounding sight as input and outputs the prediction value of the surrounding sight to evaluate the state. The value network is designed following the structure shown in Table II, where comprises two convolution-pooling layers and three FC layers. "Relu" still works as the activation function for all the layers except the last one.

TABLE II

Value Network Structure

\begin{tabular}{|c|c|c|}
\hline Layer & Filter size & stride \\
\hline Conv2D-16 & $5 \times 5$ & $1 \times 1$ \\
\hline Max_pooling2D & $2 \times 2$ & $2 \times 2$ \\
\hline Conv2D-64 & $3 \times 3$ & $1 \times 1$ \\
\hline Max_pooling2D & $2 \times 2$ & $2 \times 2$ \\
\hline \multicolumn{3}{|c|}{ FC-256 } \\
\hline \multicolumn{3}{|c|}{ FC-64 } \\
\hline & FC-1 \\
\hline
\end{tabular}

Structure of the landing spot generator described in Section III-D is shown in Table III. The landing spot generator takes a

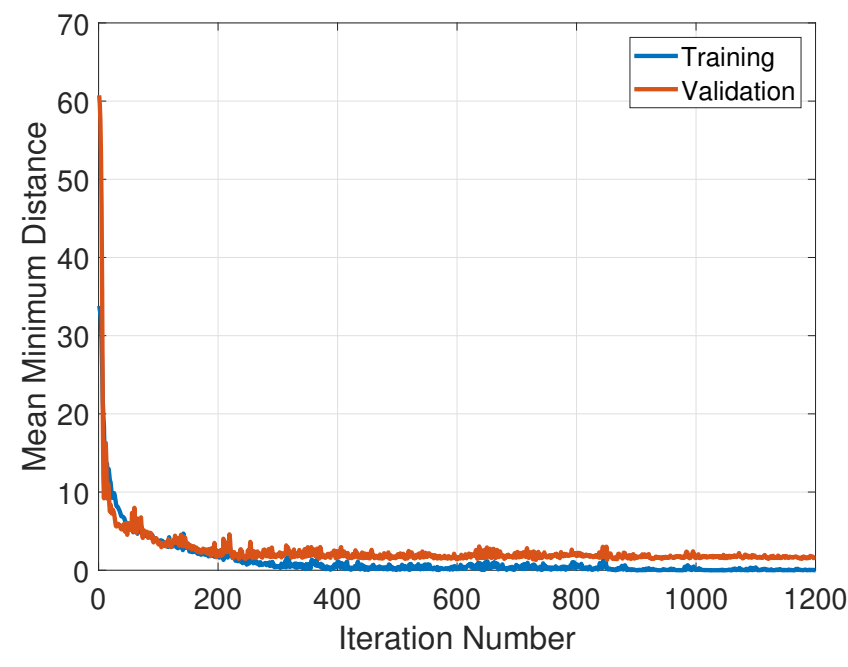

Fig. 3. Training process of the landing spot generator.

sub-image with uniform size $100 \times 80$ as input and output the predicted coordinate of the agent's landing spot through three convolution-pooling layers followed by three fully connected (FC) layers. "Relu" is selected as the activation function for all the layers except the last one.

TABLE III

Structure OF LANDING SPOT GENERATOR

\begin{tabular}{|c|c|c|}
\hline Layer & Filter size & stride \\
\hline Conv2D-32 & $5 \times 5$ & $1 \times 1$ \\
\hline Max_pooling2D & $2 \times 2$ & $2 \times 2$ \\
\hline Conv2D-64 & $5 \times 5$ & $1 \times 1$ \\
\hline Max_pooling2D & $2 \times 2$ & $2 \times 2$ \\
\hline Conv2D-64 & $5 \times 5$ & $1 \times 1$ \\
\hline Max_pooling2D & $2 \times 2$ & $2 \times 2$ \\
\hline \multicolumn{3}{|c|}{ FC-1024 } \\
\hline \multicolumn{3}{|c}{ FC-64 } \\
\hline \multicolumn{3}{|c}{ FC-2 }
\end{tabular}

\section{Landing Spot}

In this subsection, we evaluate the performance of the proposed landing spot generator. Due to the limited training set of 32 cardiac images, data augmentation is first carried out by flipping each sub-image and its associated contour up-sidedown such that the total number of training data is doubled to 64 . The upper-right sub-image of each cardiac image with size $100 \times 80$ is taken as the input of the landing spot generator. The training process with 1200 iterations is shown is Fig. 3 . Although the entire training set instead of only a batch is fed into the model, the decreasing trend in the training curve is still not stable due to the non-convexity of the loss function defined in (4). However, Algorithm 2 still works well for both training and validation sets, i.e., it converges after a few iterations of parameter update through gradient descent with the optimal 

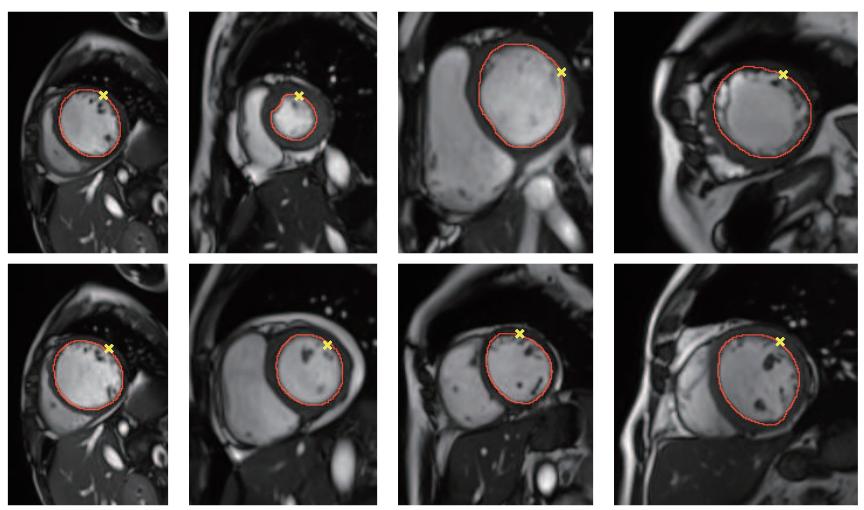

Fig. 4. Landing spots generated by Algorithm 2 for 8 randomly selected cardiac images, where the red contours are the ground-truth ones and the yellow crosses are the generated landing spot

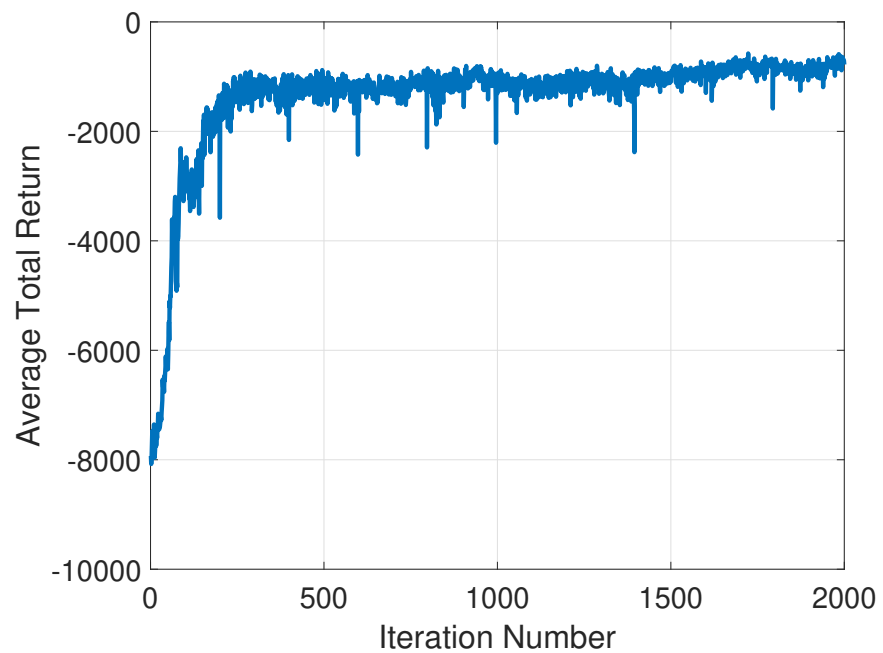

Fig. 5. Training process of the reinforcement learning model.

learning rate and generalization ability of the model keeps improving fast.

For eight cardiac images from the testing set, Fig. 4 demonstrates the agent's landing spots generated by the model specified in Table III, where the red outlines are the groundtruth contours and the yellow crosses are the generated landing spots. We can see that the the proposed landing spot generator works well after sufficient training. The coordinate of the point it generates is always sufficiently close to the true contour, which makes the generated landing spot a very good start for the following steps in left ventricle contouring.

\section{Contouring Performance}

Starting from the landing spot, the agent then sequentially makes its movement decision by following the policy network trained via Algorithm 1 to draw the contour of the left ventricle. Throughout the experiments, the agent's eyesight, i.e., the input of the policy network, is fixed at a $21 \times 21$ square patch. The extremely small negative reward to penalize the agent for moving outside a cardiac image before the episode
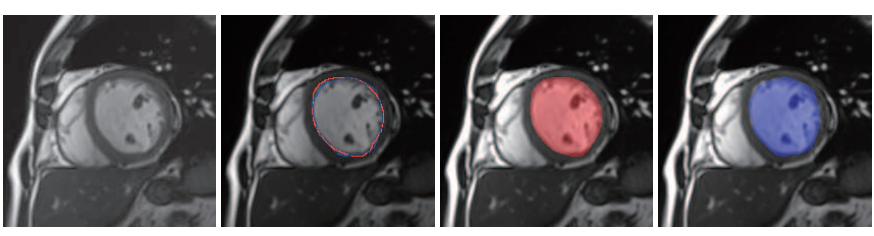

Dice Score $=0.966$
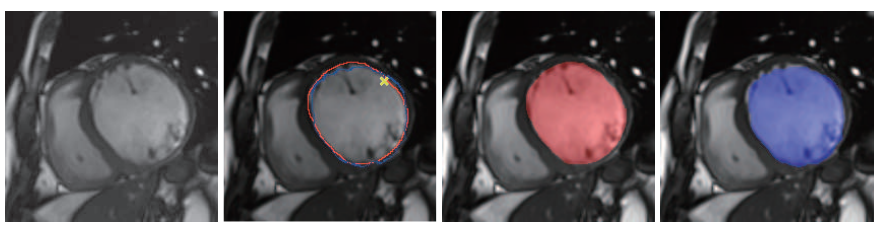

Dice Score $=0.951$
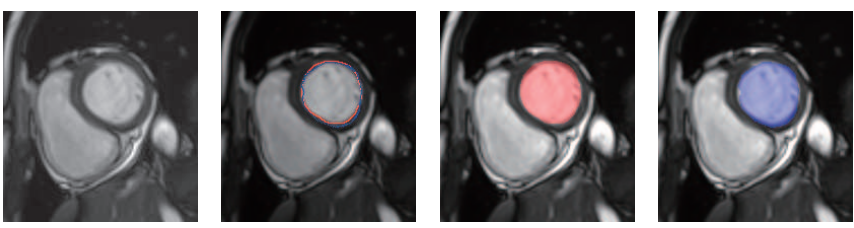

Dice Score $=0.954$
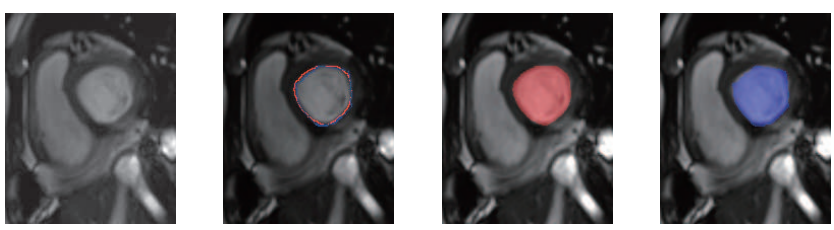

Dice Score $=0.956$
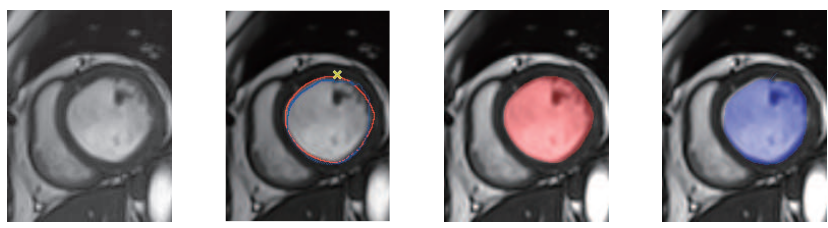

Dice Score $=0.950$
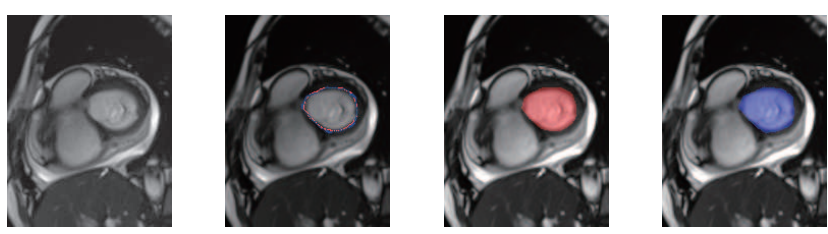

Dice Score $=0.974$
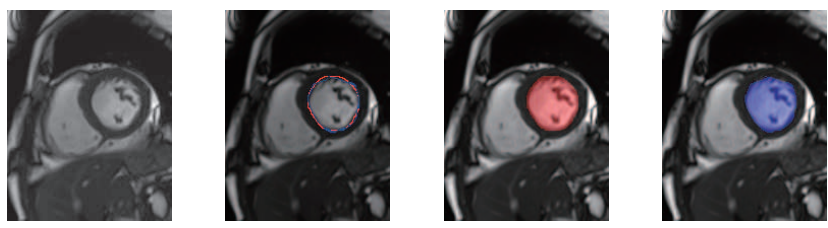

Dice Score $=0.959$
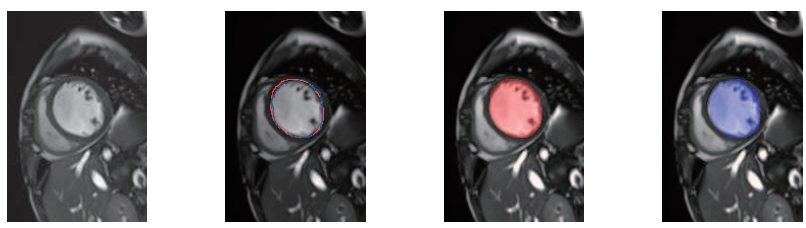

Dice Score $=0.964$

Fig. 6. Segmentation results of the left ventricle in 8 sample images based on the deep reinforcement learning method. From left to right are the example-original MR image, the ground-truth (red) and predicted (blue) contours, the ground truth segmentation and the predicted segmentation. 
ends normally is set to $-400^{1}$, and the discount factor is set to 0.997 . The whole model is trained following Algorithm 1 with adaptive moment estimation (more popularly known as "Adam") as the optimizer. Moreover, during the testing stage when the agent draws the contour on an unseen cardiac image from the testing set, episode termination indicator is still necessary to be designed since the ground-truth contour is never known by the agent. Therefore, we specify that an episode is terminated if the agent have moved into the $3 \times 3$ square patch centered by its first five positions on the contour, which indicates that the agent has finished contouring and returned to the vicinity of the landing spot (e.g., a small area within which it started) since the contour of a left ventricle is roughly the shape of a closed circle.

During model training, eight positions on the true contour are randomly selected as the agent's starting points for each cardiac image, which leads to a total of 256 experience trajectories in each iteration for the entire 32 training images. In each iteration, gradient-descent-based parameter update for policy and value networks (step 5 and 6 in Algorithm 1) is alternately carried out with each for 20 times and the learning rate is exponentially decreased during training. To improve training efficiency, we conduct min-max normalization for the reward and z-score standardization for the advantage. Moreover, the network parameters are actually stored in each iteration only when the updated parameters improve the average total return achieved with the validation set.

The training process with 2000 iterations is demonstrated is Fig. 5. In general, Algorithm 1 is able to efficiently converge. However, it is still noticeable that the training process could be temporarily "trapped" in local optimums and the total return even drops in the first 200 iterations. This is a normal case in deep reinforcement learning tasks due to the randomly sampled episode trajectories. Moreover, it can be found that the average non-discounted total return of an entire episode reaches around -570 after convergence. According to the contour images in the training set, the length of a contour amounts to 240 on average, which leads to a per-step deviation of less than 2.4 pixels on average away from the true contour. Considering the sizes of the cardiac images, this is a considerably convincing result in practice due to inevitable uncertainty and randomness in object contouring, i.e., one human expert cannot exactly duplicate a contour drawn by another one (even him-or-herself) for a same object.

We consider two metrics for contouring performance evaluation: Dice score and Hausdorff distance. Dice score is a typical performance indicator for image segmentation and is defined as

$$
\operatorname{Dice}\left(\mathcal{S}_{p}, \mathcal{S}_{g}\right)=\frac{2\left|\mathcal{S}_{p} \cap \mathcal{S}_{g}\right|}{\left|\mathcal{S}_{p}\right|+\left|\mathcal{S}_{g}\right|},
$$

where $\mathcal{S}_{p}$ and $\mathcal{S}_{g}$ denote the point (pixel) sets of the predicted and ground-truth segmentations, respectively, and |.| represents

\footnotetext{
${ }^{1}$ For the six different sizes of the cardiac images in our dataset $(208 \times 138$, $208 \times 150,208 \times 162,208 \times 168,208 \times 186$ and $208 \times 210$ ), such a penalty reward of 400 is much longer than the maximum diagonal distance of the cardiac images (less than 300 for the largest size $208 \times 210$ ), which is longer than the distance between any two points on a cardiac image. Thus, setting the penalty reward to -400 is able to sufficiently punish the agent's out-of-bound movement.
}

the cardinality of a set, i.e., the total number of pixels in the segment for this case. Hausdorff distance is an important metric for quantitatively evaluating the distance between two sets of points and thus can be defined as a performance indicator to evaluate the similarity between two contours. It is defined as

$$
d_{H}(\mathcal{G}, \mathcal{P})=\max \left\{\sup _{g \in \mathcal{G}} \inf _{p \in \mathcal{P}} d(\mathcal{G}, \mathcal{P}), \sup _{p \in \mathcal{P}} \inf _{g \in \mathcal{G}} d(\mathcal{G}, \mathcal{P})\right\},
$$

where $\mathcal{G}$ and $\mathcal{P}$ are the ground-truth and predicted contours, respectively, and $d(g, p)$ represents the Euclidean distance between two points $g$ and $p$.

With only 32 cardiac images as the training set, contouring performance is evaluated with a testing set that contains 60 images. The visual result for contouring performance evaluation with eight randomly selected testing images is shown in Fig. 6, where the corresponding segmentation results are provided as well ${ }^{2}$. Although it is difficult to ensure exactly full consistency, the contours of the left ventricle generated via the proposed approach still maintain high resemblance to the ground-truth ones. As previously discussed, these results are reasonably convincing because a radiological expert can never exactly duplicate the contour drawn previously even for the same cardiac image, which is analogous to the fact that one can never exactly duplicate his/her signature. The segmentation results shown in the last two columns of Fig. 6 also illustrate competitive performance of the proposed approach in terms of object segmentation. It is also notable that even if the predicted landing spot deviates to a certain extent from the ground-truth contour, e.g., the predicted contours in the second and fifth rows Fig. 6 as marked by the yellow crosses, the agent is still able to get back on track following the well-trained model so long as its sight covers part of the ground-truth contour. Moreover, it can be found that during testing, there could be cases that the agent temporarily "gets trapped" and wanders around within a certain area. This is because at some certain positions, the agent might be confused by the ambiguity within its sight and tends to make a random move, i.e., the output probability distribution of the policy network approximate a uniform one. However, in these cases, the agent is still able to find its way after a few steps of random movement. For this reason, the predicted contours are visually "thicker" than ground-truth ones within some certain areas.

We compare the proposed approach with the existing Unet model, which is intended for medical image segmentation based on supervised learning. The result of contouring performance comparison with the same training set of 32 images is summarized in Table IV. Here data augmentation, which triples the train set to 96 images through resizing/cropping to uniform size $208 \times 162$ with horizontal and vertical flipping, is optionally conducted for U-net model training.

According to the numerical results in Table IV, the proposed reinforcement-learning-based approach for left ventricle contouring achieves $92.6 \%$ in average Dice score and 5.784 in average Hausdorff distance, which is a satisfactory result,

\footnotetext{
${ }^{2}$ Since left ventricle contouring in cardiac images is analogized to a path finding problem, one can refer to [24] for animated contouring results.
} 
TABLE IV

Contouring Performance Comparison

\begin{tabular}{|c|c|c|}
\hline Metrics & Average Dice Score (Standard Deviation) & Average Hausdorff Distance (Standard Deviation) \\
\hline U-net without data augmentation & $0.896(0.075)$ & $14.158(13.386)$ \\
\hline U-net with data augmentation & $0.935(0.052)$ & $11.966(11.784)$ \\
\hline Ours & $0.926(0.043)$ & $5.784(1.713)$ \\
\hline
\end{tabular}
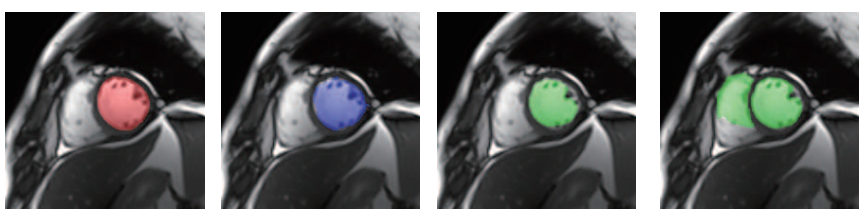

Dice Score $=0.952$
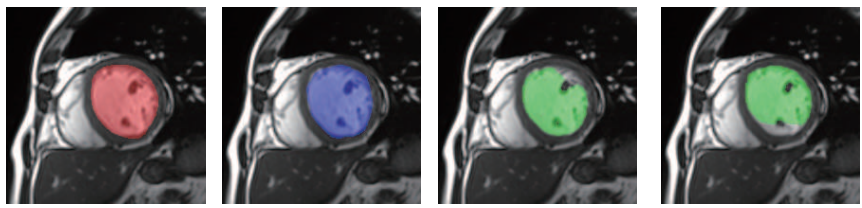

Dice Score $=0.966$
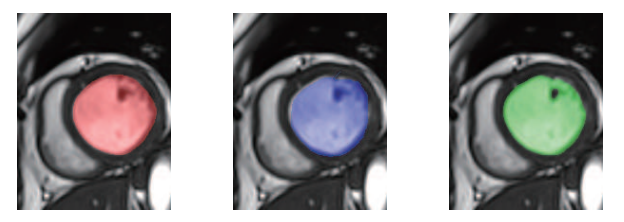

Dice Score $=0.884$

Dice Score $=0.950$
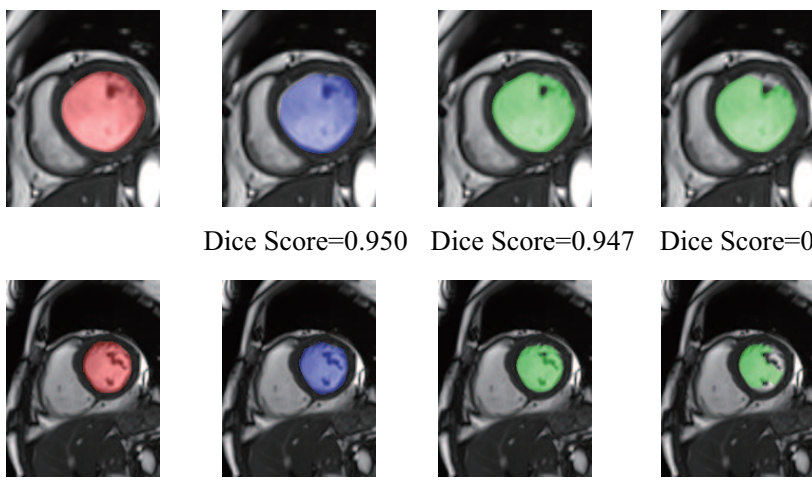

Dice Score $=0.926$

Dice Score $=0.959$ Dice Score $=0.923$ Dice Score $=0.775$

Fig. 7. Visual comparison for segmentation performance with four sample images. From left to right are the ground truth, the proposed approach, U-net with data augmentation and U-net without data augmentation.

especially considering our limited training set of only 32 images. Table IV also shows that in general, the proposed approach outperforms the U-net model with limited training set. An exception is that the proposed approach falls a little behind (while is still comparable to) the U-net model with data augmentation in average Dice score. Especially, for average Hausdorff distance, the proposed approach shows a significant advantage over the U-net model, even with data augmentation. This can be well explained by how the proposed reinforcement-learning-based approach works, i.e., it focuses on drawing the contour of an object instead of segmentation.

Fig. 7 illustrates the visual result of segmentation performance comparison on randomly selected images. The last two columns show that with limited training set, the segmentation performance of the U-net model (even with data augmentation) is less satisfactory on cardiac images with irregular cavities on the left ventricles, where unexpected "grooves" and "holes" appear in the segmentation. This is because U-net might not work well with existence of fuzzy edges that make them nondifferentiable, e.g., the left and right ventricles are located too close to be distinguished from one another (the first row in Fig. 7 as an example). In contrast, similar to Fig. 6, left ventricles identified by the proposed approach are still highly consistent with the ground-truth ones. This is still owed to the fact that the proposed reinforcement-learning-based approach aims at drawing contours as close to the ground-truth ones as possible. With well trained policy network in the proposed approach, those irregular cavities never distract the agent in its movement decision making.

\section{CONCLUSION}

In this paper, instead of focusing on computational structure of an end-to-end model for left ventricle segmentation, we focus on imitating how a human expert outlines the left ventricle in a cardiac image. Following this notion, we think of left ventricle contouring as a path finding problem and propose a novel reinforcement-learning-based framework, where the agent interacts with the unknown environment and to make the movement based on its local observation such that its trajectory matches the true contour of the left ventricle as much as possible. We also design a deep learning model and customize the loss function to generate the agent's landing spot. The experiment results show that the coordinate of the generated landing spot is sufficiently close to the true contour and the proposed reinforcement-learning-based approach outperforms the existing U-net model even with limited training set.

\section{REFERENCES}

[1] S. S. Virani, A. Alonso, H. J. Aparicio, and et al., "Heart disease and stroke statistics-2021 update: A report from the american heart association," Circulation, vol. 143, no. 8, pp. e254-e743, 2021.

[2] D. P. Ripley, T. A. Musa, L. E. Dobson, S. Plein, and J. P. Greenwood, "Cardiovascular magnetic resonance imaging: what the general cardiologist should know," Heart, vol. 102, no. 19, pp. 1589-1603, 2016. [Online]. Available: https://heart.bmj.com/content/102/19/1589

[3] J. Caudron, J. Fares, V. Lefebvre, P.-H. Vivier, C. Petitjean, and J.-N. Dacher, "Diagnostic accuracy and variability of three semi-quantitative methods for assessing right ventricular systolic function from cardiac MRI in patients with acquired heart disease," European Radiology, vol. 21, no. 10, pp. 2111-20, 2011.

[4] Y. LeCun, Y. Bengio, and G. Hinton, "Deep learning," Nature, vol. 521, no. 7553, pp. 436-444, 2015. [Online]. Available: https://doi.org/10.1038/nature14539

[5] D. Rav, C. Wong, F. Deligianni, M. Berthelot, J. Andreu-Perez, B. Lo, and G.-Z. Yang, "Deep learning for health informatics," IEEE Journal of Biomedical and Health Informatics, vol. 21, no. 1, pp. 4-21, 2017.

[6] O. Ronneberger, P. Fischer, and T. Brox, "U-net: Convolutional networks for biomedical image segmentation," in Medical Image Computing and Computer-Assisted Intervention - MICCAI 2015, N. Navab, J. Hornegger, W. M. Wells, and A. F. Frangi, Eds. Cham: Springer International Publishing, 2015, pp. 234-241.

[7] J. Long, E. Shelhamer, and T. Darrell, "Fully convolutional networks for semantic segmentation," in The IEEE Conference on Computer Vision and Pattern Recognition (CVPR), June 2015. 
[8] G. Litjens, T. Kooi, B. E. Bejnordi, A. A. A. Setio, F. Ciompi, M. Ghafoorian, J. A. van der Laak, B. van Ginneken, and C. I. Snchez, "A survey on deep learning in medical image analysis," Medical Image Analysis, vol. 42, pp. 60-88, 2017. [Online]. Available: https://www.sciencedirect.com/science/article/pii/S1361841517301135

[9] R. S. Sutton and A. G. Barto, Reinforcement Learning: An Introduction, 2nd ed. The MIT Press, 2018. [Online]. Available: http://incompleteideas.net/book/the-book-2nd.html

[10] R. C. Gonzalez and R. E. Woods, Digital Image Processing (3rd Edition). USA: Prentice-Hall, Inc., 2006.

[11] J.-1. Fan and F. Zhao, "Two-dimensional otsu's curve thresholding segmentation method for gray-level images," Acta Electronica Sinica, vol. 35, no. 4, p. 751, 2007.

[12] G. E. Hinton and R. R. Salakhutdinov, "Reducing the dimensionality of data with neural networks," science, vol. 313, no. 5786, pp. 504-507, 2006.

[13] A. Krizhevsky, I. Sutskever, and G. E. Hinton, "Imagenet classification with deep convolutional neural networks," in Advances in Neural Information Processing Systems, p. 2012.

[14] S. Zheng, S. Jayasumana, B. Romera-Paredes, V. Vineet, Z. Su, D. Du, C. Huang, and P. H. S. Torr, "Conditional random fields as recurrent neural networks," in 2015 IEEE International Conference on Computer Vision (ICCV), 2015, pp. 1529-1537.

[15] G. Lin, C. Shen, A. van den Hengel, and I. Reid, "Efficient piecewise training of deep structured models for semantic segmentation," in 2016 IEEE Conference on Computer Vision and Pattern Recognition (CVPR), 2016, pp. 3194-3203.

[16] V. Badrinarayanan, A. Kendall, and R. Cipolla, "Segnet: A deep convolutional encoder-decoder architecture for image segmentation," IEEE Transactions on Pattern Analysis and Machine Intelligence, vol. 39, no. 12, pp. 2481-2495, 2017.

[17] F. Milletari, N. Navab, and S.-A. Ahmadi, "V-net: Fully convolutional neural networks for volumetric medical image segmentation," in 2016 Fourth International Conference on 3D Vision (3DV), 2016, pp. 565571.

[18] Ö. Çiçek, A. Abdulkadir, S. S. Lienkamp, T. Brox, and O. Ronneberger, "3d u-net: Learning dense volumetric segmentation from sparse annotation," in Medical Image Computing and Computer-Assisted Intervention - MICCAI 2016, S. Ourselin, L. Joskowicz, M. R. Sabuncu, G. Unal, and W. Wells, Eds. Cham: Springer International Publishing, 2016, pp. 424-432.

[19] Z. Zhou, M. M. Rahman Siddiquee, N. Tajbakhsh, and J. Liang, "Unet++: A nested u-net architecture for medical image segmentation," in Deep Learning in Medical Image Analysis and Multimodal Learning for Clinical Decision Support, D. Stoyanov, Z. Taylor, G. Carneiro, T. Syeda-Mahmood, A. Martel, L. Maier-Hein, J. M. R. Tavares, A. Bradley, J. P. Papa, V. Belagiannis, J. C. Nascimento, Z. Lu, S. Conjeti, M. Moradi, H. Greenspan, and A. Madabhushi, Eds. Cham: Springer International Publishing, 2018, pp. 3-11.

[20] X. Li, H. Chen, X. Qi, Q. Dou, C.-W. Fu, and P.-A. Heng, "H-denseunet: hybrid densely connected unet for liver and tumor segmentation from ct volumes," IEEE transactions on medical imaging, vol. 37, no. 12, pp. 2663-2674, 2018.

[21] A.-A.-Z. Imran, A. Hatamizadeh, S. Ananth, X. Ding, N. Tajbakhsh, and D. Terzopoulos, "Fast and automatic segmentation of pulmonary lobes from chest ct using a progressive dense v-network," Computer Methods in Biomechanics and Biomedical Engineering: Imaging Visualization, vol. 8, pp. 1-10, 112019.

[22] T. Lei, W. Zhou, Y. Zhang, R. Wang, H. Meng, and A. K. Nandi, "Lightweight v-net for liver segmentation," in ICASSP 2020 - 2020 IEEE International Conference on Acoustics, Speech and Signal Processing (ICASSP), 2020, pp. 1379-1383.

[23] J. Schulman, F. Wolski, P. Dhariwal, A. Radford, and O. Klimov, "Proximal policy optimization algorithms," CoRR, vol. abs/1707.06347, 2017. [Online]. Available: http://arxiv.org/abs/1707.06347

[24] https://github.com/H1997ym/LV-contour-trajectory/. 